\title{
MASTOFAUNA DEL CERRO DE LA TUZA, OAXACA.
}

\author{
IVÁN LIRA TORRES, LAURA MORA AMBRIZ, MARCO ANTONIO CAMACHO \\ ESCOBAR Y ROSA ELENA GALINDO AGUILAR
}

Universidad del Mar - Campus Puerto Escondido (UMAR). Puerto Escondido, San Pedro Mixtepec, Juquila, Oaxaca, México. C.P.071980.ilira@zicatela.umar.mx

Resumen: Se presenta una lista de mamíferos del Cerro de la Tuza, localizado en la planicie costera del suroeste del Estado de Oaxaca. En muestreos periódicos que se llevaron a cabo de agosto del 2003 a julio del 2005, y consultas a bases de datos y publicaciones, fueron encontradas 52 especies y 22 familias en 9 órdenes. El Cerro de la Tuza que es un de los últimos remanentes de vegetación natural en la costa de Oaxaca aloja al 11\% total de especies de mamíferos en México y el 27\% para el Estado de Oaxaca.

Palabras clave: Cerro de la Tuza, Conservación, Mamíferos.

\begin{abstract}
A checklist of the mammals of the Cerro de la Tuza is presented. This area is located in the pacific coast of the State of Oaxaca. Periodical sampling periods were carried out between August 2003 andJuly 2005, 52 species, 22 families and 9 orders, of mammals were recorded. The Cerro de la Tuza is one of the last remants of natural vegetation in the Oaxaca coast. It maintains $11.52 \%$ of the total mammalian species in Mexico and the $27 \%$ of the state of Oaxaca.
\end{abstract}

Key words: Cerro de la Tuza, Conservation, Mammals.

\section{INTRODUCCIÓN}

La acelerada transformación de los ecosistemas naturales, la explotación excesiva de especies, la pérdida de la biodiversidad y la contaminación han generado una enorme crisis ambiental. El Estado de Oaxaca resalta por su riqueza animal; sin embargo también es uno de los que presentan graves problemas para su conservación. Un problema directo y complejo es la cacería sin control y el saqueo ilegal de especies para el comercio, que hacen que muchas especies de mamíferos se encuentren amenazadas de extinción. Sin embargo, existen escasos inventarios de especies presentes en regiones con remanentes de vegetación natural. Oaxaca tiene 65 especies bajo alguna categoría de protección (Briones-Salas y Sánchez-Cordero, 2004; Ceballos et al., 2002; Gonzáles et al., 2004; Webb y Baker, 1969).

Considerando que los listados de especies proveen información sólida y confiable referente a los cambios en las poblaciones de vertebrados terrestres, su 
fenología y patrones de abundancia geográfica a escala local, regional y global, en este trabajo se compiló un listado de mamíferos con datos de bases de datos de colecciones nacionales y extranjeras, publicaciones y trabajo de campo, para proponer medidas y criterios de conservación Cerro de la Tuza, un área prioritaria para la conservación del estado de Oaxaca (Droege et al., 1998).

\section{SITIO DE ESTUDIO Y MÉTODOS}

El Cerro de la Tuza, municipio de Santiago Jamiltepec, se ubica al SO del Estado de Oaxaca en la región costa, entre las coordenadas $16^{\circ} 03^{\prime} 03.0^{\prime \prime} \mathrm{N}$ y $97^{\circ} 51^{\prime} 45.0^{\prime \prime} \mathrm{O}$, limitado al sureste con el Parque Nacional Lagunas de Chacahua, al sur por el Océano Pacifico, al norte por la Sierra Madre del Sur y al oeste por el Río La Arena (Lira y Naranjo, 2005). El clima predominante es cálido subhúmedo con lluvias en verano (Awo y Aw1; García, 1973). La precipitación y temperatura varían de 500 a 1500 mm y de $22 \mathrm{a} 34^{\circ} \mathrm{C}$ respectivamente (Arriaga et al., 2000). Los tipos de vegetación dominante son selva baja caducifolia, selva mediana subcaducifolia y subperennifolia (Rzedowski, 1991; Torres, 2004; Figura 1).

Se llevaron a cabo diez viajes de campo con duración de seis días cada uno, durante el periodo de agosto del 2003 a junio del 2005. El registro de mamíferos fue a través de métodos directos e indirectos. Los métodos directos consistieron en registros visuales, auditivos y capturas. Los indirectos de huellas, restos óseos y excretas. Los individuos de talla pequeña se recolectaron con 100 trampas tipo Sherman y una mezcla de avena, extracto de vainilla, plátano y crema de cacahuate como cebo. Se colocaron en cinco transectos de 20 trampas cada uno, con una separación de 10 m entre ellas y 50 m entre transectos. Para los de talla media, se emplearon trampas tipo Tomahawk y pollos vivos como cebo. Se realizaron recorridos diurnos y nocturnos en transectos lineales de amplitud variada de 0.5 a $3.5 \mathrm{~km}$, elegidos al azar y georreferenciados en cada tipo de hábitat, en los qué se llevó el registro de huellas, excretas y observaciones directas. En algunos casos fue necesario tomar el molde de las huellas en yeso odontológico, además de contar con el registro fotográfico para su posterior identificación. Para especies de mamíferos voladores, se emplearon redes de nylon grueso de $2 \mathrm{~m}$ x $12 \mathrm{~m}$ con $3 \mathrm{~cm}$ de diámetro de luz de malla. Se colocaron entre la vegetación, caminos y cuerpos de agua. Se mantuvieron desplegadas desde las 18:00 a las 24:00 h.

Los ejemplares fueron determinados siguiendo las claves de Aranda (2000), Hall (1981), Medellín (1997), Reid (1997) y Villa (1966) tomándose los datos merísticos correspondientes. Fueron consultada bases de datos y publicaciones que pudieran tener en su acervo ejemplares del Estado de Oaxaca y de la localidad en particular, de los cuales sólo tres tuvieron información del Cerro de la Tuza.

La distribución y estado de conservación de las especies se estableció de acuerdo a la Unión Internacional para la Conservación de la Naturaleza (IUCN), la 
vol. 9
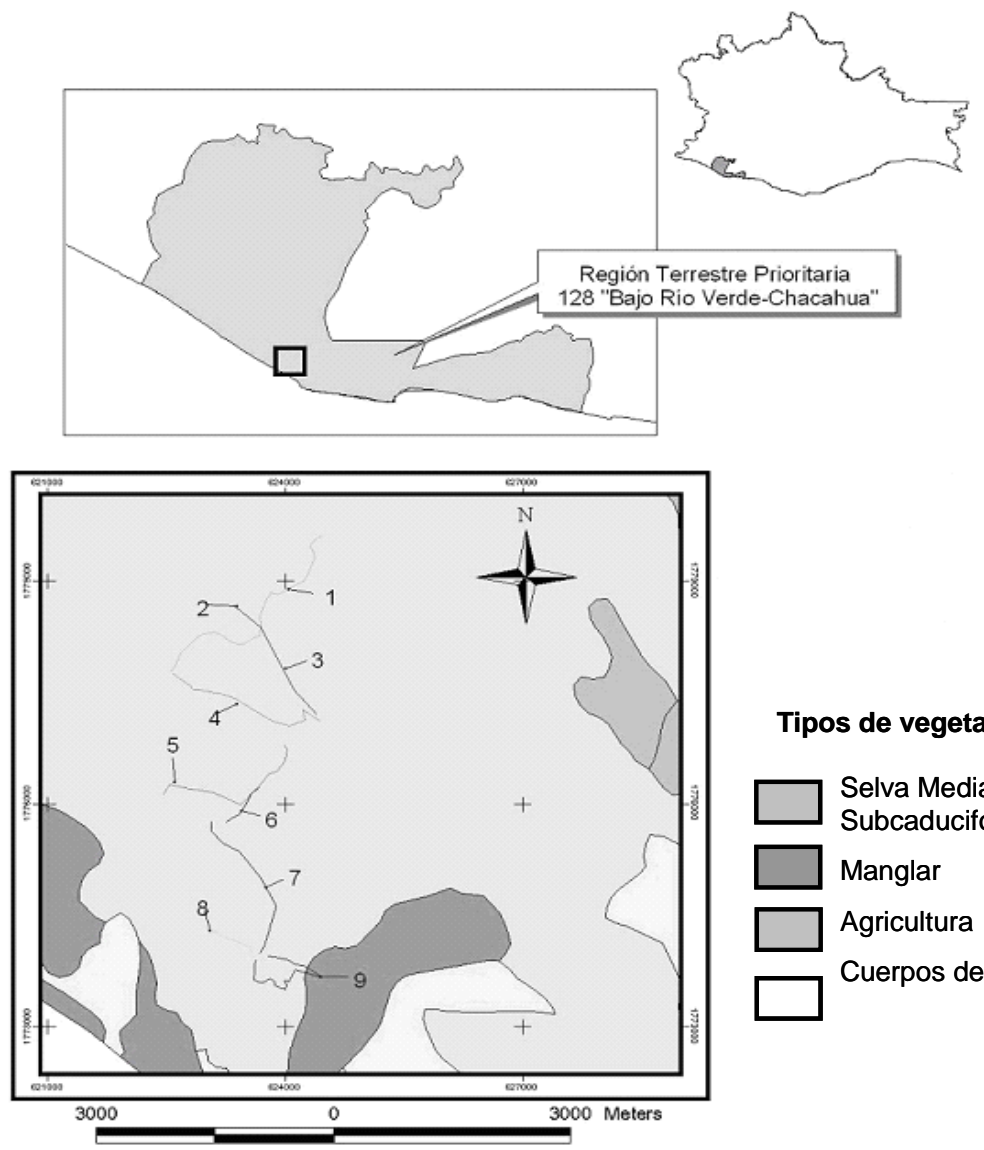

Tipos de vegetación

Selva Mediana

Subcaducifolia

$\square$ Manglar

$\square$ Agricultura

Cuerpos de Agua

Figura 1. Localización del área de estudio en el Cerro de la Tuza, Costa de Oaxaca. Los senderos con cacería persistente fueron: Casa de Piedra (1), Mixteco (5), Poza Verde (7), La Milpa (8). Los senderos con cacería moderada o baja son: Arroyo Catecuan (2), El Aguajote (3), Las Ánimas (4), sendero Tamandúa (6) y Cerro Tapír (9). 
Convención Internacional de Tráfico de especies de Flora y Fauna Silvestres (CITES) y por la Secretaria de Medio Ambiente y Recursos Naturales (Norma Oficial Mexicana SEMARNAT NOM-059-ECOL-2002, Protección Ambiental-Especies Nativas de México de Flora y Fauna Silvestre-Categoría de Riesgo y Especificaciones para su Inclusión, Exclusión o Cambio-Lista de Especies en Riesgo).

\section{RESULTADOS Y DISCUSIÓN}

La mastofauna del Cerro de la Tuza está integrada por nueve órdenes, 22 familias y 52 especies. Si se considera que el total de especies de mamíferos para México es de 522 (Ceballos et al., 2002), el Cerro de la Tuza cuenta con el 11.5\% del total nacional, y representa el 27\% del Estado de Oaxaca, mismo que cuenta con 190 especies - 42 monotípicas, 148 politípicas con 219 subespecies (Briones-Salas y Sánchez -Cordero, 2004). Con este estudio se han aportado 12 nuevos registros a los 40 que se tenia para el área. En cuanto a distribución, 18 especies (34\%) son compartidas con Sudamérica, lo que demuestra su afinidad hacia esta región. Se encuentran 13 especies (25\%) endémicas de Mesoamérica; 15 (29\%) son compartidas con Norteamérica y Sudamérica lo que demuestra que esta área mantiene el flujo de especies entre diferentes regiones; Neartica y Neotropical, en ambos sentidos, retroalimentando la diversidad mastofaunística en el sur de México. Finalmente, Spilogale pygmaea, Peromyscus melanophrys y Sylvilagus cunicularius son endémicos de México (Figura 2).

De las especies registradas en la localidad, 19 (36\%) son las consideradas por la SEMARNAT, IUCN, y CITES con algún tipo de protección (UICN, 2004; Apéndice 2). Existen 22 especies con importancia socio económica, ya sea por que son utilizados como alimento, mascotas, o por los daños generados a los cultivos de las localidades circunvecinas y debe llevarse su control. Entre los que destacan por su importancia y vulnerabilidad son: Tapirus bairdii, Leopardus pardalis, Leopardus wiedii, Puma concolor, Coendou mexicanus, Spilogale pygmaea, Lontra longicaudis, Potos flavus y Tamandua mexicana (Apéndice 3).

Entre los principales problemas de la región se encuentran la expansión de la frontera agropecuaria, los incendios forestales, la cacería de subsistencia y la creación de nuevos centros de población. En el Estado de Oaxaca se presentan dos corredores biológicos importantes para la distribución y dispersión de las especies entre Centroamérica y Norteamérica. El primero se encuentra en la Sierra Madre de Oaxaca, en donde predomina los Bosques Mesofilos de Montaña y las Selvas Altas de la vertiente del Golfo. El segundo corredor es la Costa del Pacifico en donde se encuentran varios tipos de vegetación como la selvas secas, manglares, y selvas medianas, complementándose con la Sierra Madre del Sur, con mayor número de tipos de vegetación y gran variedad de climas por los gradientes altitudinales presentes. 


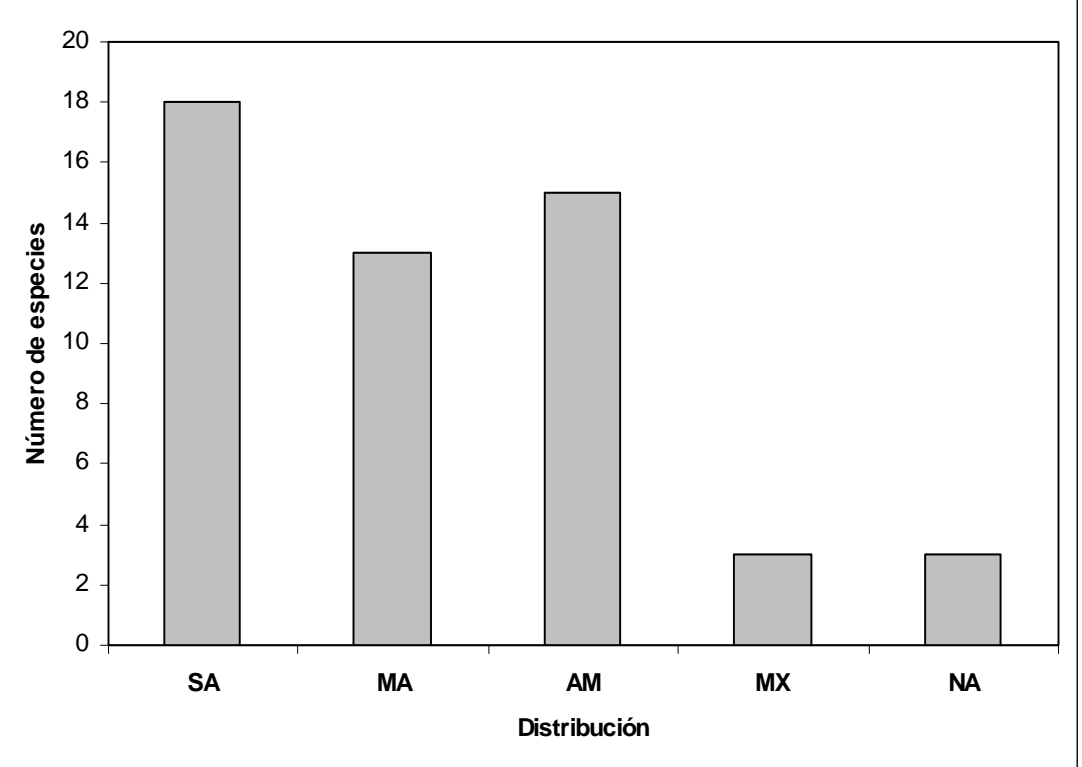

Figura 2. Total de especies de acuerdo a su distribución. SA (Compartidas con Sudamérica), MA (Endémica de Mesoamérica), AM (Compartida con Norte y Sudamérica), MX (Endémicas de México), NA (Compartida con Norteamérica).

En este sentido El Cerro de la Tuza representa un refugio único para especies que se encuentran amenazadas o en peligro de extinción, y es el último corredor biológico que conecta la Costa del Pacifico Oaxaqueño en la región Suroeste con la Sierra Madre del Sur, manteniendo el flujo genético de estas poblaciones (Torres, 2004). Cabe destacar que la lista de mamíferos que se presenta es parcial, falta seguir realizando más estudios y observaciones en el lugar, principalmente en los roedores, quirópteros e insectívoros.

\section{AGRADECIMIENTOS}

Los autores agradecen a la Universidad del Mar (UMAR) a través del Proyecto: Estatus Poblacional e Impacto de la Cacería de Subsistencia en Vertebrados del Bajo Río Verde, Oaxaca, México (Clave Interna: 21E0401), así como a la Comisión Nacional de Áreas Naturales Protegidas / Parque Nacional Lagunas de Chacahua (CONANP/ PNLCH), por el apoyo logístico brindado y el financiamiento otorgado. También agradecemos de manera muy especial al Biol. Mario Enrique Fuente Carrasco y 
M.A.I.A. Cuitláhuac Hernández Santiago, por sus valiosos comentarios y aportaciones para el desarrollo de este proyecto. A los pobladores del Ejido de la Tuza de Monroy, Municipio de Santiago Jamiltepec; en especial a la Familia Santiago Castro, por la hospitalidad y facilidades prestadas para este estudio.

\section{LITERATURACITADA}

Aranda, M. 2000. Huellas y otros rastros de los mamíferos grandes y medianos de México. Instituto de Ecología A.C., Xalapa, México.

Arriaga, L., J. M. Espinoza, C. Aguilar, E. Martínez, L. Gómez y E. Loa (eds.). 2000. Regiones terrestres prioritarias de México. Comisión Nacional para el Conocimiento y Uso de la Biodiversidad, México.

Briones-Salas, M. y V. Sánchez-Cordero. 2004. Mamíferos. Pp 423-447, en: Biodiversidad de Oaxaca. (A. J. García- Mendoza, M. J. Ordóñez y M. Briones-Salas, eds.), Instituto de Biología, UNAM, Fondo Oaxaqueño para la Conservación de la Naturaleza-Word Wildlife Fund, México.

Ceballos, G., J. Arroyo-Cabrales, y R. A. Medellín. 2002. Mamíferos de México. Pp. 378413, en: Diversidad y Conservación de los Mamíferos Neotropicales. (G. Ceballos y J. A. Simonetti, eds.). 2002. CONABIO-UNAM. México, D.F.

Droege, S., A. Cyr, y J. Larivee. 1998. Chechlists: An Under-Used Tood for the Inventory and Monitoring of Plants and Animals. Conservation Biology, 12:1134-1138.

García, E. 1973. Modificaciones al sistema de clasificación climática de Köppen. Instituto de Geografía, U.N.A.M. México.

Gonzáles-Pérez, G., M. Briones-Salas y A. M. Alfaro. 2004. Integración del conocimiento faunístico del estado. Pp. 449-466, en: Biodiversidad de Oaxaca. (A. J. García - Mendoza, M. J. Ordóñez y M. Briones-Salas, eds.), Instituto de Biología, UNAM, Fondo Oaxaqueño para la Conservación de la Naturaleza - Word Wildlife Fund, México.

Hall, E. R. 1981. The Mammals of North America. Vols. I, II. John Wiley \& Sons. New York, EUA.

IUCN 2004. 2004 IUCN Red List of Threatened Species. Gland, Suiza.

Lira, T. I., y E. Naranjo, P. 2005. Ampliación del área de distribución de Tapirus bairdii Gill 1865 (Perisodactila: Tapiridae) en México. Acta Zoológica Mexicana (nueva serie), 21:107110.

Medellín, R. A., H. T. Arita y O. Sánchez T. 1997. Identificación de los murciélagos de México: Clave de campo. Publicaciones Especiales, Asociación Mexicana de Mastozoología, A.C.

Reid, A. F. 1997. A Field guide to the mammals of central and southeast Mexico. Oxford University Press, Nueva York.

Rzedowsky, J. 1991. Vegetación de México. Limusa. México.

Secretaría de Medio Ambiente, Recursos Naturales y Pesca (SEMARNAP). 2002. Norma Oficial Mexicana NOM-059-ECOL-2000. Protección ambiental, especies de flora y fauna silvestres de México, categorías de riesgo y especificaciones para su inclusión, exclusión o cambio, y lista de especies en riesgo. Diario Oficial de la Federación, Lunes 16 de octubre de 2001, 1:1-62. 
Torres Colín, R. 2004. Tipos de Vegetación. Pp. 105 - 117, en: Biodiversidad de Oaxaca. (A. J. García-Mendoza, M. J. Ordóñez y M. Briones-Salas, eds.), Instituto de Biología, UNAM, Fondo Oaxaqueño para la Conservación de la Naturaleza-Word Wildlife Fund, México.

Villa, R. B. 1966. Los Murciélagos de México. Instituto de Biología, UNAM., México, D.F. Webb, R.G. y R. H. Baker. 1969. Vertebrados terrestres del suroeste de Oaxaca. Anales del Instituto de Biología, Universidad Nacional Autónoma de México, Serie Zoología, 40(1):139-152.

Wilson, D. E. y D. M. Reeder (eds.). 1993. Mammals Species of the World, a taxonomic and geographic reference. 2nd ed. Smithsonian Institution Press, Washington, D. C. 


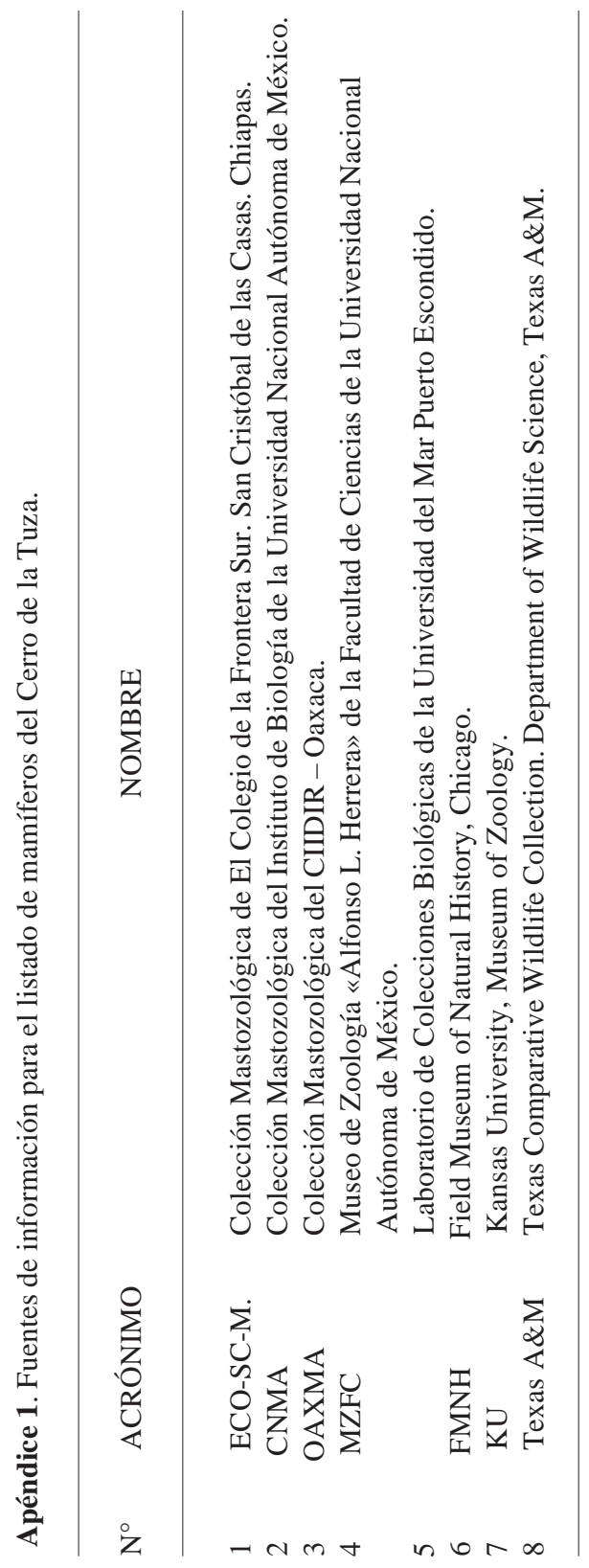



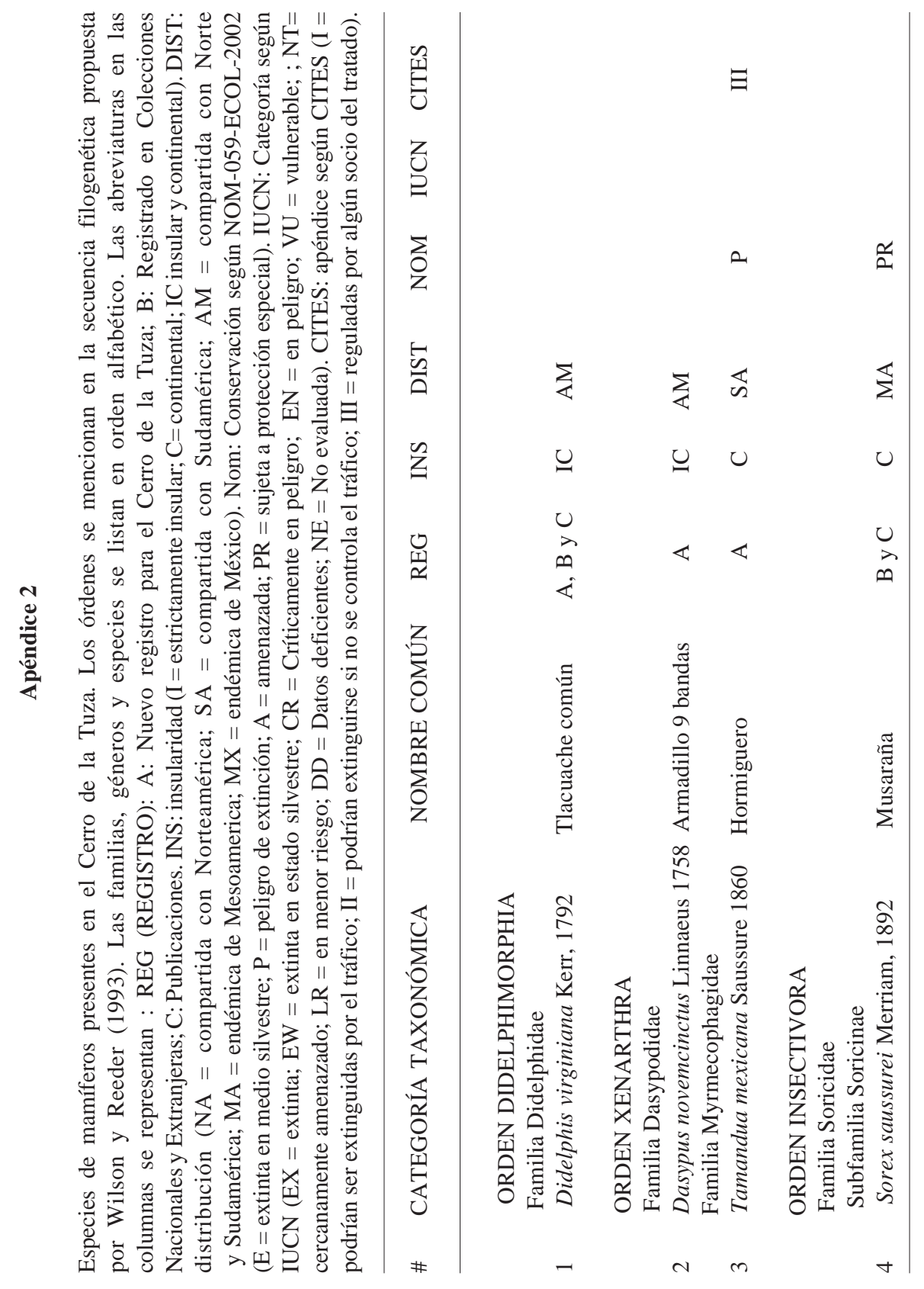


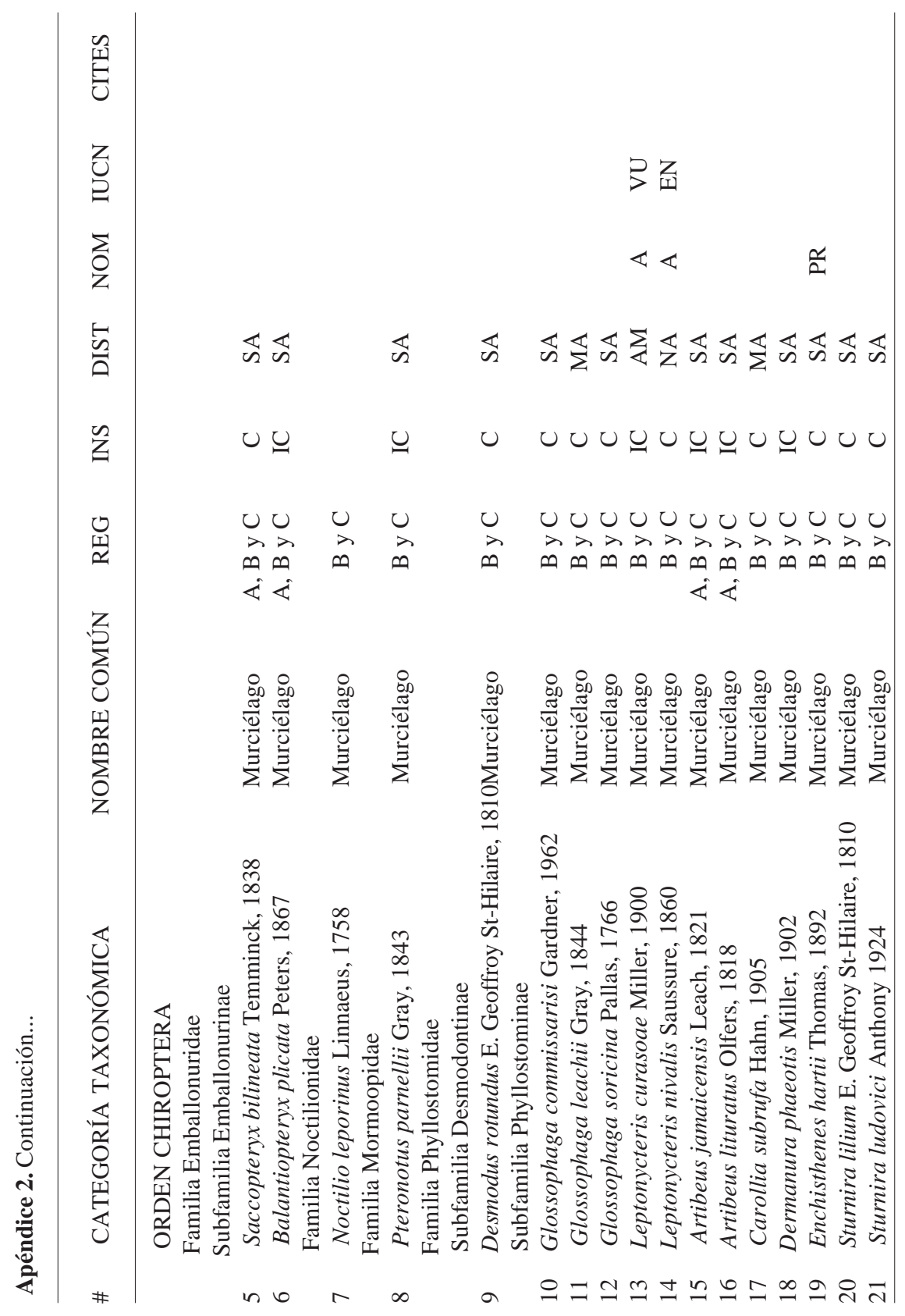




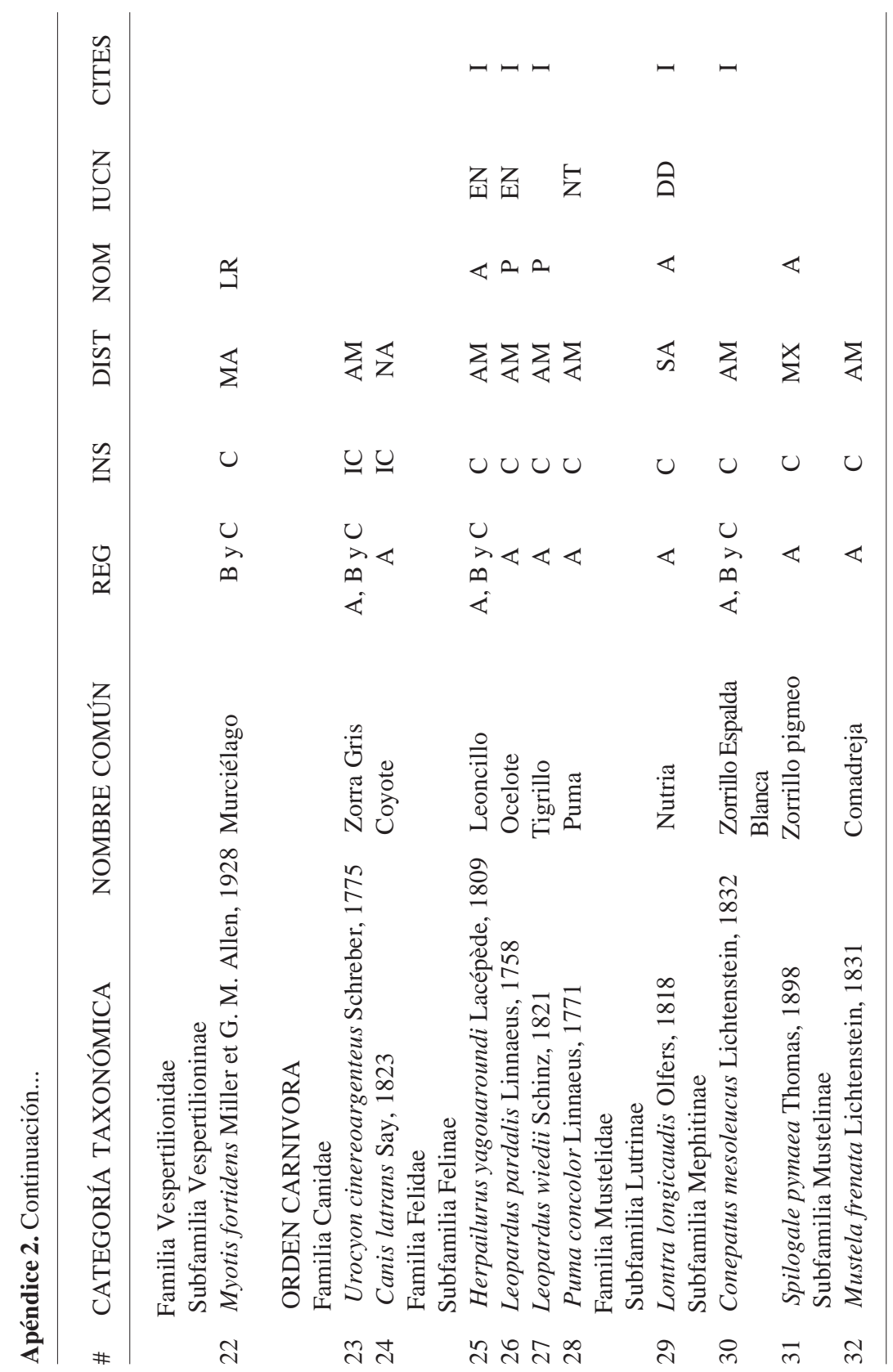




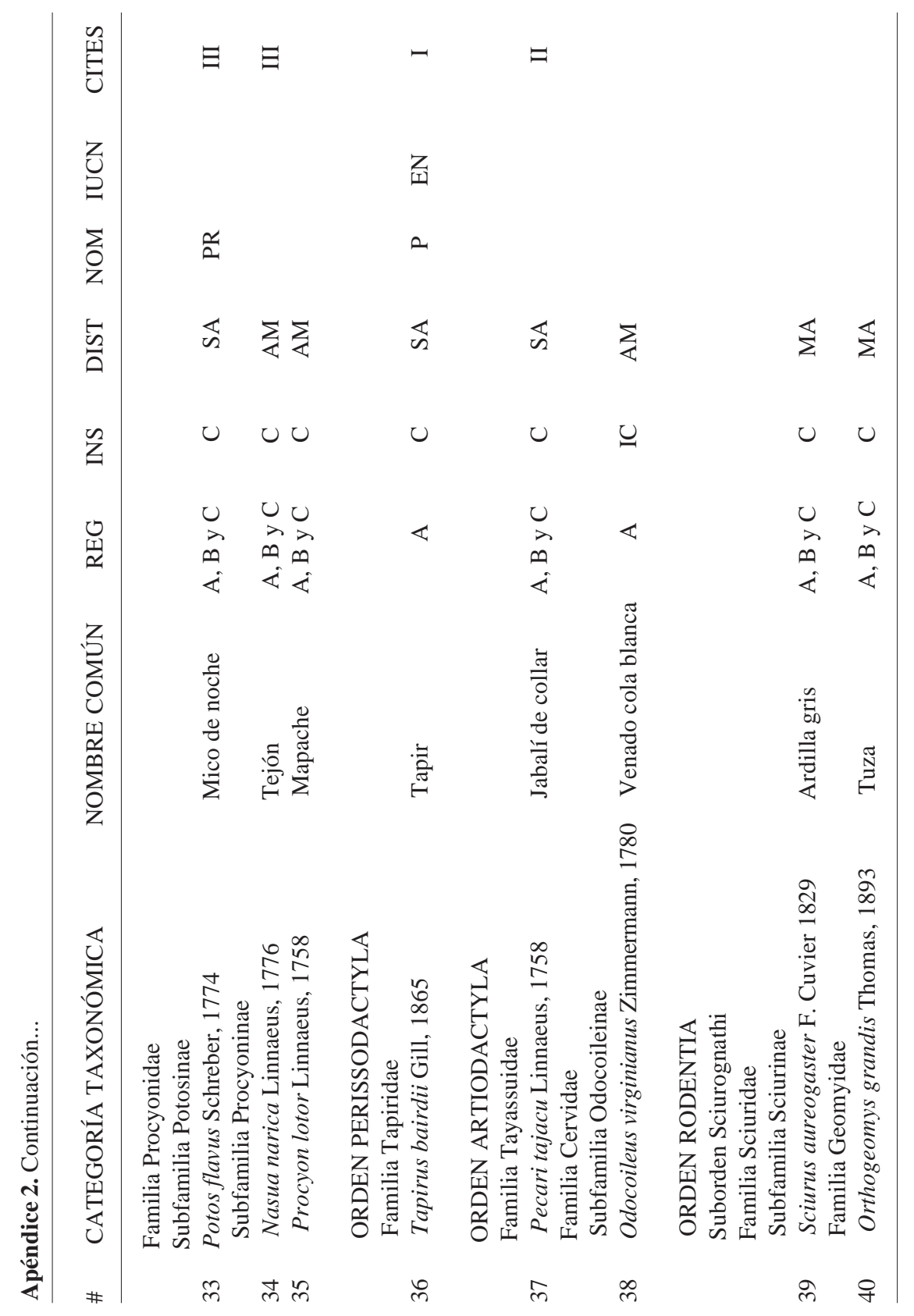




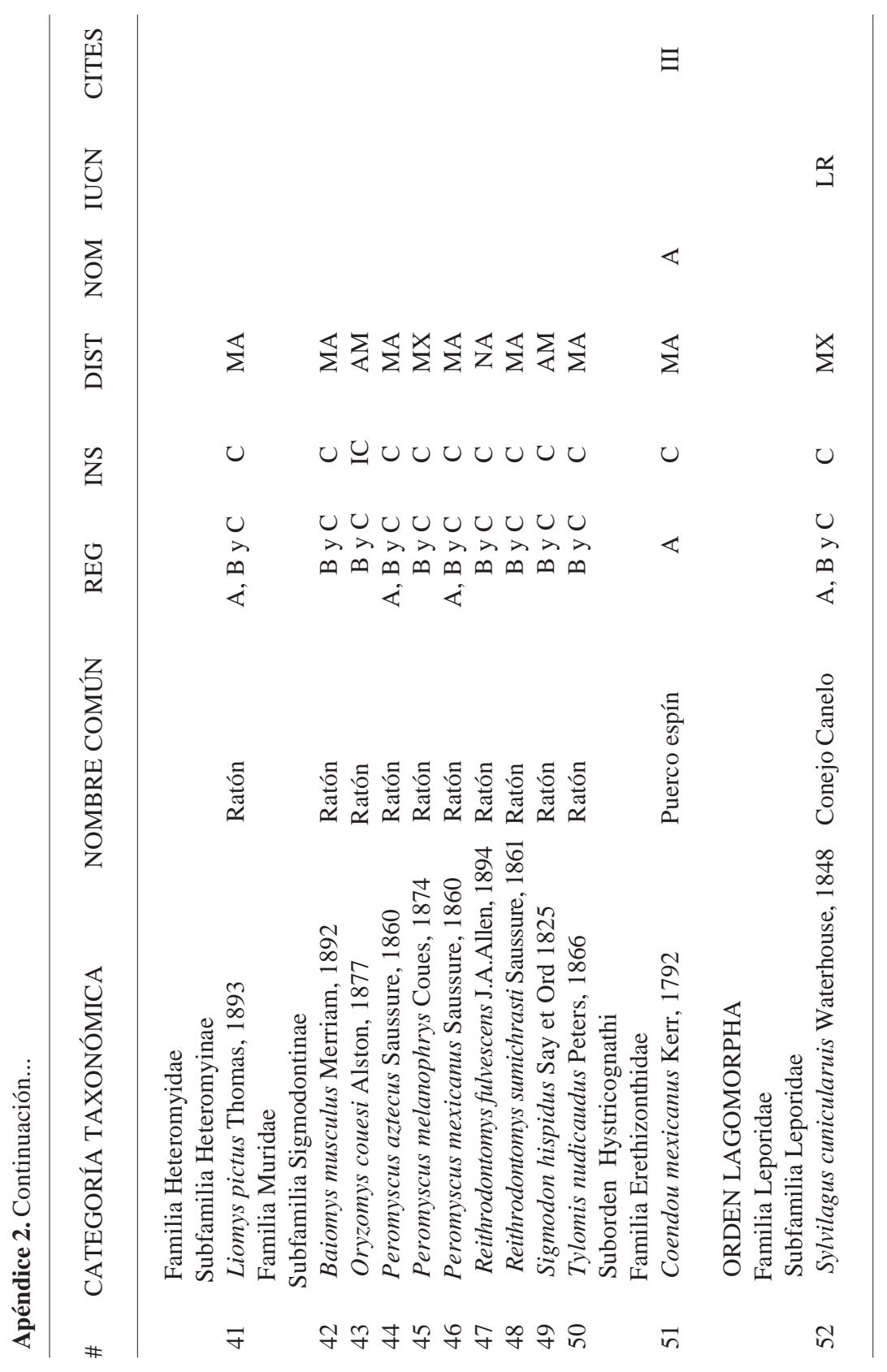




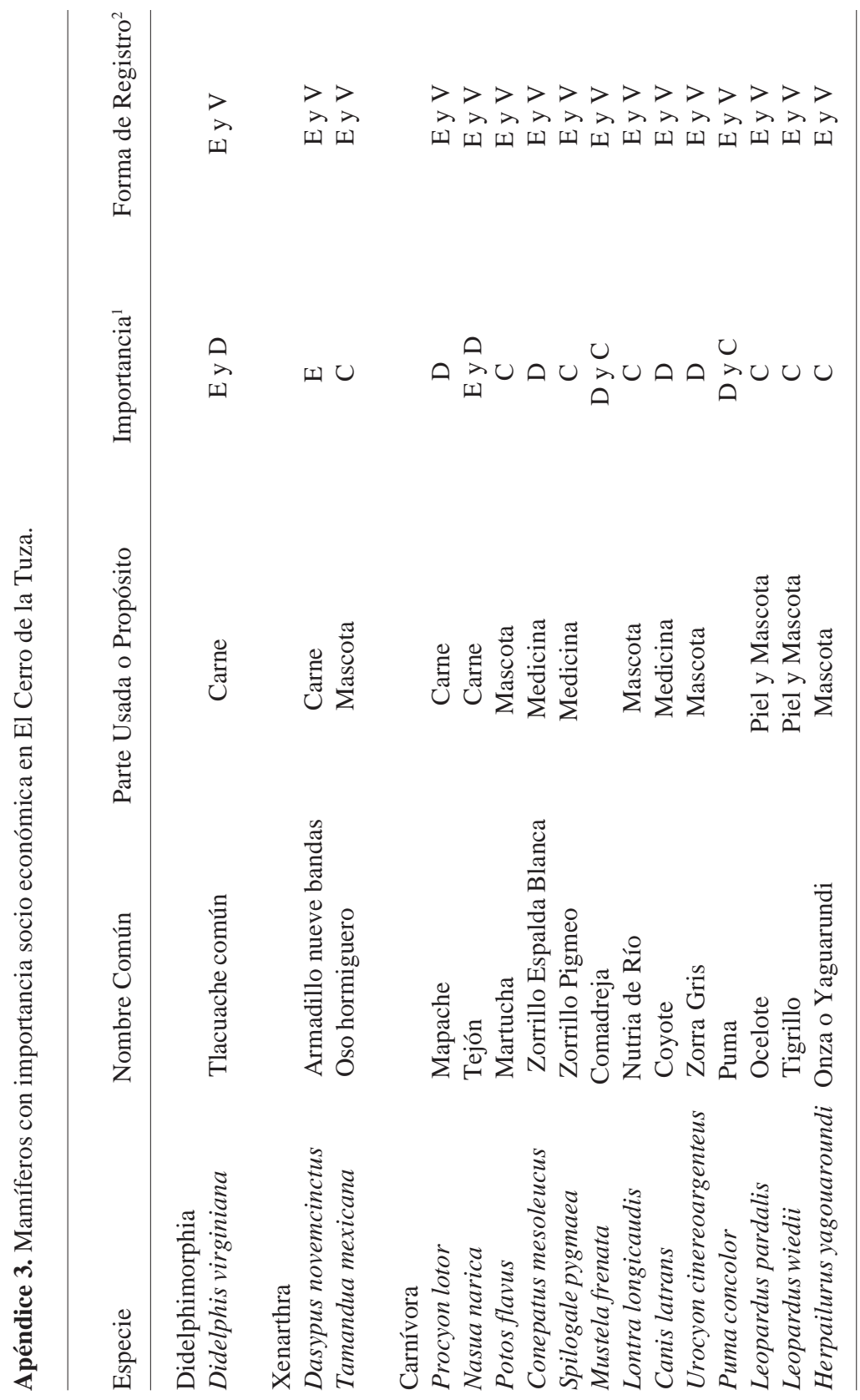


vol. 9

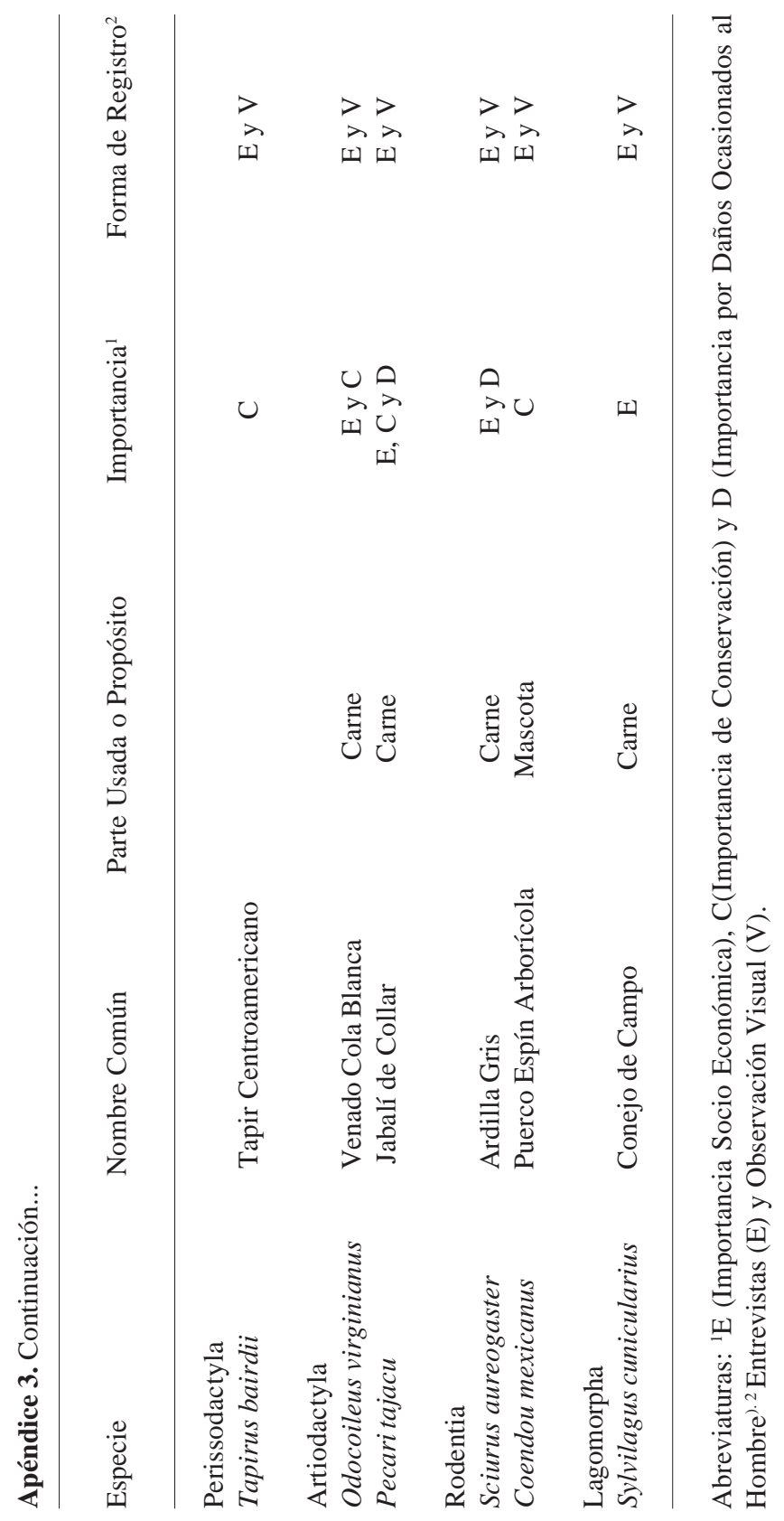

